\title{
A UNIFIED APPROACH TO THE HEAVY-TRAFFIC ANALYSIS OF THE MAXIMUM OF RANDOM WALKS*
}

\author{
S. SHNEER ${ }^{\dagger}$ AND V. WACHTEL ${ }^{\ddagger}$ \\ (Translated by the authors)
}

\begin{abstract}
For families of random walks $\left\{S_{k}^{(a)}\right\}$ with $\mathbf{E} S_{k}^{(a)}=-k a<0$ we consider their maxima $M^{(a)}=\sup _{k \geqq 0} S_{k}^{(a)}$. We investigate the asymptotic behavior of $M^{(a)}$ as $a \rightarrow 0$ for random walks from the domain of attraction of a stable law. This problem appeared first in the 1960s in the analysis of a single-server queue when the traffic load tends to 1 , and since then it is referred to as the heavy-traffic approximation problem. Kingman and Prokhorov suggested two different approaches which were later followed by many authors. We give two elementary proofs of our main result, using each of these approaches. It turns out that the main technical difficulties in both proofs are rather similar and may be resolved via a generalization of the Kolmogorov inequality to the case of an infinite variance. Such a generalization is also obtained in this paper.
\end{abstract}

Key words. random walk, global maximum, stable law, Kolmogorov inequality generalization

DOI. $10.1137 /$ S0040585X97984796

Assume that $\left\{X_{i}\right\}_{i=1}^{\infty}$ is a sequence of independent identically distributed random variables with zero expectation $\mathbf{E} X_{1}=0$. Define a random walk

$$
S_{0}=0, \quad S_{k}=\sum_{i=1}^{k} X_{i} \quad \text { for } \quad k \geqq 1 .
$$

Along with the random walk $\left\{S_{k}\right\}$, for each $a>0$ define a random walk $\left\{S_{k}^{(a)}\right\}$ via

$$
S_{k}^{(a)}=S_{k}-k a .
$$

Now we can define

$$
M^{(a)}=\sup _{k \geqq 0} S_{k}^{(a)}
$$

Since random walk $S_{k}^{(a)}$ drifts to $-\infty$, the maximum $M^{(a)}$ is a proper random variable for each $a>0$. However, $M^{(a)} \rightarrow+\infty$ in probability as $a \rightarrow 0$. From this fact the following natural question arises: How fast does $M^{(a)}$ grow as $a \rightarrow 0$ ?

It is well known that a stationary distribution of the waiting time of a customer in a single-server first-come-first-served $(G I / G I / 1)$ queue coincides with that of the maximum of a corresponding random walk. The condition on the mean of the random walk to get small $(a \rightarrow 0)$ means in the context of a queue that the traffic load tends to 1 . Thus, the problem under consideration may be seen as an investigation of the growth rate of the stationary waiting-time distribution in a $G I / G I / 1$ queue. This is one of the most important problems in queueing theory and is referred to as heavy-traffic analysis. The question was first posed by Kingman [7], [8] (see also [9] for an extensive discussion) who considered the case when $\left|X_{1}\right|$ has an exponential moment and proved that

$$
\mathbf{P}\left(a M^{(a)} \leqq x\right) \rightarrow 1-e^{-2 x / \sigma^{2}}
$$

\footnotetext{
* Received by the editors December 3, 2009.

http://www.siam.org/journals/tvp/55-2/98479.html

†Sobolev Institute of Mathematics SBRAS, Academician Koptiug Pr. 4, 630090 Novosibirsk, Russia (sevasneer@ngs.ru).

${ }^{\ddagger}$ Weierstrass Institute for Applied Analysis and Stochastics, Mohrenstr., 39, Berlin, Germany, and Technische Universität München, Boltzmann str., 3, München, Germany (wachtel@ma.tum.de, vakhtel@wais-berlin.de).
} 
for all $x \geqq 0$ as $a \rightarrow 0$, where $\sigma^{2}=\operatorname{Var} X_{1}$. In his proof, Kingman used an analytical approach. Namely, he applied an expression for the Laplace transform of $M^{(a)}$ which follows from the Wiener-Hopf factorization.

Prokhorov [10] generalized the latter result to for case when $\operatorname{Var} X_{1} \in(0, \infty)$. Prokhorov's approach was based on the functional central limit theorem.

These two approaches have become classical and have both been used to prove various heavy-traffic results. However, they seem to have never been compared. Later in this paper we shall discuss both approaches in more detail and point out their differences and similarities. The analytical approach was used by Boxma and Cohen [3] (see also [4]) to study the limiting behavior of $M^{(a)}$ in the case of infinite variance. They proved that if $\mathbf{P}\left(X_{1}>x\right)$ is regularly varying at infinity with a parameter $1<\alpha<2$ (and under some additional assumptions), then there exists a function $\Delta(a)$ such that $\Delta(a) M^{(a)}$ converges in distribution to a proper random variable. Furrer [5] and Resnick and Samorodnitsky [12] proved similar results assuming that the random walk $\left\{S_{n}\right\}$ belongs to the domain of attraction of a spectrally positive stable distribution and using functional limit theorems. It is worth mentioning that Furrer has computed the corresponding limit distribution explicitly. Borovkov in his recent paper [2] proved the convergence of $\Delta(a) M^{(a)}$ for any random walk from the domain of attraction of a stable law with index $\alpha \in(1,2)$. His proof also relies on functional limit theorems.

The main purpose of the present paper is to suggest a unified way of studying the asymptotic behavior of $M^{(a)}$. In other words, we provide a method that works for any random walk from the domain of attraction of a stable law.

A random walk $\left\{S_{n}\right\}$ is said to belong to the domain of attraction of a stable law with index $\alpha \in(0,2]$ if there exist sequences $b_{n}$ and $c_{n}$ such that

$$
\frac{S_{n}-b_{n}}{c_{n}} \rightarrow \xi \quad \text { weakly }
$$

where $\xi$ has the corresponding stable distribution.

It is known that the random walk $\left\{S_{n}\right\}$ belongs to the domain of attraction of a stable law with index $\alpha \in(0,2]$ if and only if the function

$$
V(x):=\mathbf{E}\left(X_{1}^{2} ;\left|X_{1}\right| \leqq x\right), \quad x>0,
$$

is regularly varying at infinity with index $2-\alpha$. The latter implies that we can choose

$$
c_{n}:=\inf \left\{u>0: \frac{V(u)}{u^{2}} \leqq \frac{1}{n}\right\}, \quad n \geqq 1 .
$$

In this case the sequence $\left\{c_{n}\right\}$ is regularly varying with index $1 / \alpha$ and, furthermore,

$$
\frac{V\left(c_{n}\right)}{c_{n}^{2}} \sim \frac{1}{n}, \quad n \rightarrow \infty
$$

In this paper we consider the case when $\left\{S_{n}\right\}$ belongs to the domain of attraction of a stable law with index $\alpha \in(1,2]$ and $\mathbf{E} X_{1}=0$. It is known that under these assumptions we can choose $b_{n} \equiv 0$. Hence, we have the convergence

$$
\frac{S_{n}}{c_{n}} \rightarrow \xi \quad \text { weakly. }
$$

Let $\left\{\xi_{t}, t \geq 0\right\}$ denote a stable Lévy process, where $\xi_{1}$ is equal in distribution to $\xi$. We also need to define the supremum of the process $\left\{\xi_{t}-t\right\}_{t \geqq 0}$ via

$$
M^{*}=\sup _{t \geqq 0}\left\{\xi_{t}-t\right\}
$$

which is a proper random variable as $\xi_{t}-t$ drifts to $-\infty$ as $t \rightarrow \infty$. 
For any $\alpha \in(1,2]$ one can choose a positive integer-valued function $n(a)$ such that

$$
\operatorname{an}(a) \sim c_{n(a)} \quad \text { as } a \rightarrow 0 .
$$

It follows from the regular variation of $\left\{c_{n}\right\}$ that $n(a)$ is regularly varying at zero with index $-\alpha /(\alpha-1)$. This, in turn, implies that $c_{n(a)}$ is regularly varying with index $-1 /(\alpha-1)$.

TheOREM. Suppose that $\left\{S_{n}\right\}$ belongs to the domain of attraction of a stable law with index $\alpha \in(1,2]$. Then, as $a \rightarrow 0$,

$$
\frac{M^{(a)}}{c_{n(a)}} \rightarrow M^{*} \quad \text { in distribution. }
$$

The theorem implies that $M^{(a)}$ grows as a regularly varying at zero function with index $-1 /(\alpha-1)$. The limit distribution (that of $\left.M^{*}\right)$ is only known explicitly in two particular cases. If $\left\{\xi_{t}\right\}$ has no positive jumps, then one has an exponential distribution. If $\left\{\xi_{t}\right\}$ has no negative jumps and $\alpha<2$, then $M^{*}$ has a Mittag-Leffler distribution (see, e.g., [5]). In the other cases an explicit form of the distribution is unknown; however, one can easily find its tail asymptotics $\mathbf{P}\left(M^{*}>x\right) \sim C x^{1-\alpha}$ as $x \rightarrow \infty$.

This result is the only new one in the case when $\alpha=2$ and $\mathbf{E} X_{1}^{2}=\infty$, and all other cases may be found in the literature mentioned at the beginning of the introduction. As we have already mentioned, our motivation consists of providing a unified proof for all asymptotically stable random walks. We will in fact give two different proofs of the theorem, with the use of both classical methods mentioned above. However, we would like to start by giving a brief description and comparison of the two methods.

The first, analytical, method is based on the analysis of the Laplace transform of the normalized maximum of a random walk. The idea is to use a corollary of the Wiener-Hopf factorization:

$$
\mathbf{E} e^{-\mu M^{(a)} / c_{n(a)}}=\exp \left\{-\sum_{k=1}^{\infty} \frac{1}{k} \mathbf{E}\left(1-e^{-\mu S_{k}^{(a)} / c_{n(a)}} ; S_{k}^{(a)}>0\right)\right\}, \quad \mu \geqq 0 .
$$

In [9] a heuristic argument is given stating that the main contribution to the infinite series in the exponent on the RHS is due to the values of $k$ of order $n(a)$. This, however, was not proved formally. Instead, the author represented the exponent in the form of an integral along the imaginary axis and gave a proof of the statement by solving a Wiener-Hopf boundaryvalue problem. The same method was used later in [3] in the case of an infinite variance.

Our proof justifies the heuristic argument of Kingman. The main difficulty consists of showing that the contribution to the infinite series on the RHS of the latter equality from values of $k \gg n(a)$ is negligible. We will show that it follows from

$$
\lim _{T \rightarrow \infty} \sum_{k \geqq T n(a)} \frac{1}{k} \mathbf{P}\left(S_{k}^{(a)}>0\right)=0
$$

uniformly in $a>0$. Thus, the derivation of (4) is a key point in our proof.

We now turn to the second method used in the literature, namely the method based on functional limit theorems. Consider the maximum of $\left\{S_{n}^{(a)}\right\}$ on a finite-time interval

$$
M_{T}^{(a)}=\sup _{k \leqq T n(a)} S_{k}^{(a)} .
$$

It easily follows from the functional limit theorems for asymptotically stable random walks that

for any $x \geqq 0$, where

$$
\lim _{a \rightarrow 0} \mathbf{P}\left(\frac{M_{T}^{(a)}}{c_{n(a)}} \geqq x\right)=\mathbf{P}\left(M_{T}^{*} \geqq x\right)
$$

$$
M_{T}^{*}=\sup _{t \leqq T}\left\{\xi_{t}-t\right\} .
$$


The latter convergence implies that

$$
\lim _{T \rightarrow \infty} \lim _{a \rightarrow 0} \mathbf{P}\left(\frac{M_{T}^{(a)}}{c_{n(a)}} \geqq x\right)=\mathbf{P}\left(M^{*} \geqq x\right) .
$$

However, in order to prove the theorem, one needs to show that

$$
\lim _{a \rightarrow 0} \lim _{T \rightarrow \infty} \mathbf{P}\left(\frac{M_{T}^{(a)}}{c_{n(a)}} \geqq x\right)=\mathbf{P}\left(M^{*} \geqq x\right) .
$$

Therefore, it remains to justify the interchange of limits. It is easy to see that a sufficient condition could be written as

$$
\lim _{T \rightarrow \infty} \mathbf{P}\left(\sup _{k \geqq n(a) T} S_{k}^{(a)} \geqq 0\right)=0
$$

uniformly in $a>0$. This was shown by Prokhorov [10] in the case of a finite variance by applying the classical Kolmogorov inequality. Later, Asmussen [1, p. 289] proved the same result using the fact that the sequence $\left\{S_{n} / n\right\}$ is a backward martingale. This fact was also utilized by Furrer [5] and Resnick and Samorodnitsky [12] in the case of an infinite variance.

It is worth mentioning that relations (4) and (5) are equivalent under the conditions of the theorem. A proof of this fact will be given at the end of this paper.

We now state and prove a generalization of the Kolmogorov inequality when the variance is not necessarily finite. This inequality allows one to overcome the technical difficulties in both approaches described above, namely, to prove (4) and (5).

Lemma. There exists $C>0$ such that the inequality

$$
\mathbf{P}\left(\max _{k \leqq n} S_{k} \geqq x\right) \leqq C \frac{n V(x)}{x^{2}}
$$

holds for all $x>0$ and $n \geqq 1$.

Proof. According to inequality (1.2) in [11],

$$
\mathbf{P}\left(\max _{k \leqq n} S_{k} \geqq x\right) \leqq C n\left(\mathbf{P}\left(\left|X_{1}\right|>x\right)+x^{-1}\left|\mathbf{E}\left(X_{1} ;\left|X_{1}\right| \leqq x\right)\right|+x^{-2} V(x)\right)
$$

(here and in what follows, $C$ denotes a generic positive and finite constant).

It is easy to see that

$$
\begin{aligned}
\mathbf{P}\left(\left|X_{1}\right|>x\right) & =\sum_{j=0}^{\infty} \mathbf{P}\left(\left|X_{1}\right| \in\left(2^{j} x, 2^{j+1} x\right]\right) \leqq \sum_{j=0}^{\infty} \frac{V\left(2^{j+1} x\right)}{2^{2 j} x^{2}} \\
& \leqq \frac{V(x)}{x^{2}} \cdot 4 C(\gamma) \sum_{j=1}^{\infty} 2^{-(\alpha-\gamma) j}
\end{aligned}
$$

where in the last step we used the inequality

$$
\frac{V(y)}{V(x)} \leqq C(\gamma)\left(\frac{y}{x}\right)^{2-\alpha+\gamma}, \quad y \geqq x,
$$

which follows from the Karamata representation; see [15, Theorem 1.2] (recall that $V(x)$ is regularly varying with index $2-\alpha$ ). Choosing $\gamma<\alpha$, we get

$$
\mathbf{P}\left(\left|X_{1}\right|>x\right) \leqq C \frac{V(x)}{x^{2}}
$$

In a way similar to that used in obtaining (9), we can get the bound

$$
\left|\mathbf{E}\left(X_{1} ;\left|X_{1}\right| \leqq x\right)\right|=\left|\mathbf{E}\left(X_{1} ;\left|X_{1}\right|>x\right)\right| \leqq C \frac{V(x)}{x} .
$$


Combining (7), (9), and (10), we get inequality (6). The proof is thus complete.

Proof of the theorem via Wiener-Hopf. The Wiener-Hopf factorization implies that

$$
\mathbf{E} e^{-\lambda M^{(a)}}=\exp \left\{\sum_{k=1}^{\infty} \frac{1}{k} \mathbf{E}\left(e^{-\lambda S_{k}^{(a)}}-1 ; S_{k}^{(a)}>0\right)\right\}
$$

for any $\lambda>0$; see, e.g., [16, Proposition 19.2]. For the purpose of our proof, we set $\lambda=$ $\mu / c_{n(a)}$ where $\mu>0$. In these terms the latter equality takes the form

$$
\mathbf{E} e^{-\mu M^{(a)} / c_{n(a)}}=\exp \left\{-\sum_{k=1}^{\infty} \frac{1}{k} \mathbf{E}\left(1-e^{-\mu S_{k}^{(a)} / c_{n(a)}} ; S_{k}^{(a)}>0\right)\right\} .
$$

We aim to show that a pointwise limit as $a \rightarrow 0$ of this Laplace transform is equal to the Laplace transform of $M^{*}$.

Fix $\varepsilon \in(0,1), T>1$ and divide the sum in the exponent in (11) into three parts:

$$
\sum_{k=1}^{\infty}=\sum_{k=1}^{\varepsilon n(a)}+\sum_{k=\varepsilon n(a)}^{T n(a)}+\sum_{k=T n(a)}^{\infty}=: \Sigma_{1}+\Sigma_{2}+\Sigma_{3}
$$

We will now analyze these three summands separately.

It follows from the inequalities $0 \leqq 1-e^{-t} \leqq t$ for any positive $t$ that

$$
0 \leqq \mathbf{E}\left(1-e^{-\mu S_{k}^{(a)} / c_{n(a)}} ; S_{k}^{(a)}>0\right) \leqq \frac{\mu}{c_{n(a)}} \mathbf{E}\left(S_{k}^{(a)} ; S_{k}^{(a)}>0\right) .
$$

Note that $\mathbf{E}\left(S_{k}^{(a)} ; S_{k}^{(a)}>0\right)=\mathbf{E}\left(S_{k}-k a ; S_{k}>k a\right) \leqq \mathbf{E}\left(S_{k} ; S_{k}>0\right)$. Since $S_{n}$ is asymptotically stable with $\alpha>1$,

$$
\lim _{n \rightarrow \infty} c_{n}^{-1} \mathbf{E}\left(S_{n} ; S_{n}>0\right)=\mathbf{E}\left(\xi_{1} ; \xi_{1}>0\right) .
$$

This implies that $\mathbf{E}\left(S_{k} ; S_{k}>0\right) \leqq C c_{k}$ for all $k \geq 1$. As a result we have the bound

$$
0 \leqq \mathbf{E}\left(1-e^{-\mu S_{k}^{(a)} / c_{n(a)}} ; S_{k}^{(a)}>0\right) \leqq C \mu \frac{c_{k}}{c_{n(a)}} .
$$

Hence,

$$
0 \leqq \Sigma_{1} \leqq \frac{C \mu}{c_{n(a)}} \sum_{k=1}^{\varepsilon n(a)} \frac{c_{k}}{k}
$$

Since the sequence $\left\{c_{k}\right\}$ is regularly varying with index $1 / \alpha \in(0,1)$,

$$
\sum_{k=1}^{n} \frac{c_{k}}{k} \sim \frac{c_{n}}{\alpha} \quad \text { as } n \rightarrow \infty
$$

Consequently,

$$
\sum_{k=1}^{\varepsilon n(a)} \frac{c_{k}}{k} \leqq C c_{\varepsilon n(a)} \leqq C \varepsilon^{1 / \alpha} c_{n(a)}
$$

Estimates (12) and (13) imply

$$
0 \leqq \Sigma_{1} \leqq C \varepsilon^{1 / \alpha} \mu
$$

In order to bound $\Sigma_{3}$ we note that

$$
0 \leqq \mathbf{E}\left(1-e^{-\mu S_{k}^{(a)} / c_{n(a)}} ; S_{k}^{(a)}>0\right) \leqq \mathbf{P}\left(S_{k}^{(a)}>0\right)=\mathbf{P}\left(S_{k}>k a\right) .
$$

Copyright (C) by SIAM. Unauthorized reproduction of this article is prohibited. 
Using the lemma, we obtain

$$
0 \leqq \Sigma_{3} \leqq \sum_{k=T n(a)}^{\infty} \frac{1}{k} \mathbf{P}\left(S_{k}>k a\right) \leqq C \sum_{k=T n(a)}^{\infty} \frac{V(k a)}{(k a)^{2}} \leqq C \frac{1}{a} \int_{\operatorname{Tan}(a)}^{\infty} \frac{V(x)}{x^{2}} d x .
$$

Recalling that $V(x)$ is regularly varying with index $2-\alpha$, we continue with

$$
\Sigma_{3} \leqq C \frac{V(\operatorname{Tan}(a))}{T a^{2} n(a)} \leqq C T^{1-\alpha} \frac{V(a n(a))}{a^{2} n(a)} \leqq C T^{1-\alpha} .
$$

In the last step we used the relations

$$
\frac{V(a n(a))}{a^{2} n(a)} \sim \frac{V\left(c_{n(a)}\right)}{\left(c_{n(a)}\right)^{2}} n(a) \sim 1, \quad a \rightarrow 0,
$$

which follow from (1).

It now remains to analyze $\Sigma_{2}$. Using the assumption that $S_{k} / c_{k}$ converges in distribution to $\xi_{1}$, we get

$$
\frac{S_{k}^{(a)}}{c_{n(a)}}=\frac{S_{k}}{c_{n(a)}}-\frac{k a}{c_{n(a)}}=\frac{S_{k}}{c_{k}} \frac{c_{k}}{c_{n(a)}}-\frac{k a}{c_{n(a)}} \longrightarrow v^{1 / \alpha} \xi_{1}-v
$$

in distribution as $a \rightarrow 0$ and $k / n(a) \rightarrow v \in(0, \infty)$. In the last step we used (2) and the regular variation of $\left\{c_{k}\right\}$. It follows from the scaling property of the stable process $\left\{\xi_{t}\right\}$ that $v^{1 / \alpha} \xi_{1}$ and $\xi_{v}$ are equal in distribution. From this relation and (17) we conclude that

$$
\mathbf{E}\left(1-e^{-\mu S_{k}^{(a)} / c_{n(a)}} ; S_{k}^{(a)}>0\right) \longrightarrow \mathbf{E}\left(1-e^{-\mu\left(\xi_{v}-v\right)} ; \xi_{v}-v>0\right)
$$

as $a \rightarrow 0$ and $k / n(a) \rightarrow v \in(0, \infty)$. Using now the dominated convergence we see that, as $a \rightarrow 0$,

$$
\begin{aligned}
\Sigma_{2} & =\sum_{k=\varepsilon n(a)}^{T n(a)} \frac{n(a)}{k} \mathbf{E}\left(1-e^{-\mu S_{k}^{(a)} / c_{n(a)}} ; S_{k}^{(a)}>0\right) \frac{1}{n(a)} \\
& \longrightarrow \int_{\varepsilon}^{T} \frac{1}{v} \mathbf{E}\left(1-e^{-\mu\left(\xi_{v}-v\right)} ; \xi_{v}-v>0\right) d v .
\end{aligned}
$$

It is clear that the process $\xi_{t}-t$ drifts to $-\infty$. Then, using [14, Theorem 48.1], we obtain

$$
\begin{aligned}
& \lim _{\varepsilon \rightarrow 0, T \rightarrow \infty} \int_{\varepsilon}^{T} \frac{1}{v} \mathbf{E}\left(1-e^{-\mu\left(\xi_{v}-v\right)} ; \xi_{v}-v>0\right) d v \\
& =\int_{0}^{\infty} \frac{1}{v} \mathbf{E}\left(1-e^{-\mu\left(\xi_{v}-v\right)} ; \xi_{v}-v>0\right) d v<\infty
\end{aligned}
$$

Hence, combining (11), (14), (15), and (18) and letting $\varepsilon \rightarrow 0, T \rightarrow \infty$, we get

$$
\mathbf{E} e^{-\mu M^{(a)} / c_{n(a)}} \longrightarrow \exp \left\{-\int_{0}^{\infty} \frac{1}{v} \mathbf{E}\left(1-e^{-\mu\left(\xi_{v}-v\right)} ; \xi_{v}-v>0\right) d v\right\}
$$

The latter expression is known to be the Laplace transform of $M^{*}$; see again [14, Theorem 48.1]. This completes the proof.

Proof of the theorem via functional limit theorems. According to the functional limit theorem for asymptotically stable random walks (see, for example, Theorem 9.6.2 of [6]), we get

$$
\left\{\frac{S_{[n t]}}{c_{n}} ; t \in[0 ; T]\right\} \rightarrow\left\{\xi_{t} ; t \in[0 ; T]\right\}
$$

Copyright (C) by SIAM. Unauthorized reproduction of this article is prohibited. 
in distribution. Therefore, as $a \downarrow 0$,

$$
\left\{\frac{S_{[n(a) t]}-[a n(a) t]}{c_{n(a)}} ; t \in[0 ; T]\right\} \longrightarrow\left\{\xi_{t}-t ; t \in[0 ; T]\right\}
$$

This convergence implies that

$$
\lim _{a \downarrow 0} \mathbf{P}\left(\max _{k \leqq n(a) T} S_{k}^{(a)} \geqq x c_{n(a)}\right)=\mathbf{P}\left(\sup _{t \leqq T}\left(\xi_{t}-t\right) \geqq x\right), \quad x \geqq 0 .
$$

Since $\sup _{t \leqq T}\left(\xi_{t}-t\right)$ converges to $M^{*}$, the theorem will be proved if we show that

$$
\lim _{T \rightarrow \infty} \mathbf{P}\left(\max _{k \geqq n(a) T} S_{k}^{(a)} \geqq 0\right)=0
$$

uniformly in $a>0$.

Note that

$$
\begin{aligned}
\left\{\max _{k \geqq n(a) T} S_{k}^{(a)} \geqq 0\right\} & =\bigcup_{j=0}^{\infty}\left\{\max _{k \in\left[2^{j} n(a) T, 2^{j+1} n(a) T\right)}\left(S_{k}-k a\right) \geqq 0\right\} \\
& \subset \bigcup_{j=0}^{\infty}\left\{\max _{k \geqq 2^{j+1} n(a) T} S_{k} \geqq 2^{j} a n(a) T\right\} .
\end{aligned}
$$

From this relation and the lemma we obtain

$$
\begin{aligned}
& \mathbf{P}\left(\max _{k \geqq n(a) T} S_{k}^{(a)} \geqq 0\right) \leqq \sum_{j=0}^{\infty} \mathbf{P}\left(\max _{k \leqq 2^{j+1} n(a) T} S_{k} \geqq 2^{j} a n(a) T\right) \\
& \quad \leqq C \sum_{j=0}^{\infty} \frac{2^{j+1} n(a) T V\left(2^{j} a n(a) T\right)}{\left(2^{j} a n(a) T\right)^{2}}=\frac{2 C}{a} \sum_{j=0}^{\infty} \frac{V\left(2^{j} a n(a) T\right)}{2^{j} a n(a) T} .
\end{aligned}
$$

With the use of (8) with some $\gamma<\alpha-1$ the latter expression can be estimated by $C V(a n(a) T) /\left(a^{2} n(a) T\right)$. The regular variation of $V(x)$ and the relation (16) yield the bound $V(a n(a) T) /\left(a^{2} n(a) T\right) \leqq C T^{1-\alpha}$, which implies (20), completing our proof.

To conclude, we would like to make a few remarks. First, we note that our assumption on the family of random walks can be weakened. We assumed that $S_{k}^{(a)}=S_{k}-k a$ for the transparency of all proofs. However, our argument remains valid if we assume, for instance, that

$$
S_{k}^{(a)}=S_{k}-k a+\sum_{i=1}^{k} Y_{i}^{(a)}
$$

where $\left\{Y_{i}^{(a)}\right\}$ is a sequence of independent identically distributed random variables such that $\mathbf{E} Y_{1}^{(a)}=0$ and $\mathbf{E}\left|Y_{1}^{(a)}\right|^{\gamma}<\infty$ uniformly in $a$ for some $\gamma>\alpha$. Indeed, one can easily verify that, uniformly in $a$,

$$
\frac{S_{n}^{(a)}+n a}{c_{n}} \rightarrow \xi \quad \text { weakly }
$$

and, furthermore,

$$
\mathbf{E}\left(\left(S_{1}^{(a)}+a\right)^{2} ;\left|S_{1}^{(a)}+a\right| \leqq x\right) \leqq C V(x),
$$

with the latter bound allowing us to apply the lemma to the random walk $\left\{S_{n}^{(a)}+n a\right\}$.

Moreover, in the case of finite variance, for our argument to remain valid, it is sufficient that a Lindeberg-type condition holds, i.e.,

$$
S_{k}^{(a)}=\sum_{i=1}^{k} X_{i}^{(a)}
$$

Copyright $@$ by SIAM. Unauthorized reproduction of this article is prohibited. 
where $\left\{X_{i}^{(a)}\right\}$ is a sequence of independent identically distributed random variables such that $\mathbf{E} X_{1}^{(a)}=-a, \lim _{a \rightarrow 0} \operatorname{Var} X_{1}^{(a)}=\sigma^{2} \in(0, \infty)$, and

$$
\lim _{a \rightarrow 0} \mathbf{E}\left(\left(X_{1}^{(a)}\right)^{2} ;\left|X_{1}^{(a)}\right|>\frac{\varepsilon}{a}\right)=0 \quad \text { for all } \varepsilon>0 .
$$

The latter condition seems to be optimal. On one hand, it is necessary, according to the Lindeberg theorem, for the normal approximation of $a S_{1 / a^{2}}^{(a)}$. On the other hand, Sakhanenko [13] gave an example of a family $S^{(a)}$ such that (21) fails and $M^{(a)}=0$ for all $a$.

Our last remark concerns the equivalence of relations (4) and (5).

Proof of the equivalence of (4) and (5). We start by showing that (5) is equivalent to the relation

$$
\lim _{T \rightarrow \infty} \mathbf{P}\left(M^{(a)}>T c_{n(a)}\right)=0 \quad \text { uniformly in } a>0 .
$$

To this end we first note that

$$
\mathbf{P}\left(\sup _{k \geqq n(a) T} S_{k}^{(a)} \geqq 0\right) \leqq \mathbf{P}\left(S_{T n(a)}^{(a)} \geqq-\operatorname{Tan}(a) / 2\right)+\mathbf{P}\left(M^{(a)}>T c_{n(a)} / 2\right)
$$

and

$$
\mathbf{P}\left(\sup _{k \geqq n(a) T} S_{k}^{(a)} \geqq 0\right) \geqq \mathbf{P}\left(M^{(a)}>2 T c_{n(a)}\right)-\mathbf{P}\left(S_{T n(a)}^{(a)} \geqq-2 \operatorname{Tan}(a)\right) .
$$

It follows from our lemma that the probabilities $\mathbf{P}\left(S_{T n(a)}^{(a)} \geqq-\operatorname{Tan}(a) / 2\right)$ and $\mathbf{P}\left(S_{T n(a)}^{(a)} \geqq\right.$ $-2 \operatorname{Tan}(a))$ converge, as $T \rightarrow \infty$, to zero uniformly in $a$. Hence, recalling that an $(a) \sim c_{n(a)}$, we get the equivalence of (5) and (22).

It is easy to see that

$$
\begin{aligned}
\mathbf{P}\left(M^{(a)} \leqq T c_{n(a)}\right) & \geqq \mathbf{E}\left[\exp \left\{-\mu \frac{M^{(a)}}{c_{n(a)}}\right\} ; M^{(a)} \leqq T c_{n(a)}\right] \\
& \geqq \mathbf{E} \exp \left\{-\mu \frac{M^{(a)}}{c_{n(a)}}\right\}-e^{-\mu T} \mathbf{P}\left(M^{(a)}>T c_{n(a)}\right) .
\end{aligned}
$$

Putting here $\mu=T^{-1}$, we have the inequality

$$
\mathbf{P}\left(M^{(a)}>T c_{n(a)}\right) \leqq \frac{1-\mathbf{E} \exp \left\{-M^{(a)} /\left(T c_{n(a)}\right)\right\}}{1-e^{-1}} .
$$

Using (14) with $\varepsilon=T$ and $\mu=T^{-1}$, we obtain

$$
\sum_{k=1}^{\infty} \frac{1}{k} \mathbf{E}\left(1-e^{-S_{k}^{(a)} /\left(T c_{n(a)}\right)} ; S_{k}^{(a)}>0\right) \leqq C T^{1 / \alpha-1}+\sum_{k=T n(a)}^{\infty} \frac{1}{k} \mathbf{P}\left(S_{k}^{(a)}>0\right) .
$$

From this bound we infer that (4) yields

$$
\lim _{T \rightarrow \infty} \sum_{k=1}^{\infty} \frac{1}{k} \mathbf{E}\left(1-e^{-S_{k}^{(a)} /\left(T c_{n(a)}\right)} ; S_{k}^{(a)}>0\right)=0 \quad \text { uniformly in } a>0 .
$$

Therefore, from (23) and (11) we conclude that (4) implies (22).

In order to prove the reverse implication we use an obvious inequality

$$
\mathbf{P}\left(M^{(a)} \leqq T c_{n(a)}\right) \leqq e^{\mu T} \mathbf{E} \exp \left\{-\mu \frac{M^{(a)}}{c_{n(a)}}\right\} .
$$

Choosing $\mu=T^{-2}$ and letting $T \rightarrow \infty$, one can easily see that (22) implies that

$$
\lim _{T \rightarrow \infty} \log \mathbf{E} \exp \left\{-\frac{M^{(a)}}{T^{2} c_{n(a)}}\right\}=0 \quad \text { uniformly in } a>0 .
$$

Copyright (c) by SIAM. Unauthorized reproduction of this article is prohibited. 
With the use of (11), we have

$$
\lim _{T \rightarrow \infty} \sum_{k=T^{6} n(a)}^{\infty} \frac{1}{k} \mathbf{E}\left(1-e^{-S_{k}^{(a)} /\left(T^{2} c_{n(a)}\right)} ; S_{k}^{(a)}>0\right)=0 \quad \text { uniformly in } a>0 .
$$

Noting that

$$
\mathbf{E}\left(1-e^{-S_{k}^{(a)} /\left(T^{2} c_{n(a)}\right)} ; S_{k}^{(a)}>0\right) \geqq\left(1-e^{-1}\right) \mathbf{P}\left(S_{k}^{(a)}>T^{2} c_{n(a)}\right),
$$

we get

$$
\lim _{T \rightarrow \infty} \sum_{k=T^{6} n(a)}^{\infty} \frac{1}{k} \mathbf{P}\left(S_{k}^{(a)}>T^{2} c_{n(a)}\right)=0 \quad \text { uniformly in } a>0 .
$$

Since the density of any stable law is bounded, it follows from the local limit theorems for $S_{n}$ that $\mathbf{P}\left(S_{n} \in[0, z)\right) \leqq C(z+1) / c_{n}$ for any $z>0$. Hence,

$$
\mathbf{P}\left(S_{k}^{(a)}>T^{2} c_{n(a)}\right) \geqq \mathbf{P}\left(S_{k}^{(a)}>0\right)-C \frac{T^{2} c_{n(a)}}{c_{k}} .
$$

Consequently,

$$
\begin{aligned}
\sum_{k=T^{6} n(a)}^{\infty} \frac{1}{k} \mathbf{P}\left(S_{k}^{(a)}>T^{2} c_{n(a)}\right) & \geqq \sum_{k=T^{6} n(a)}^{\infty} \frac{1}{k} \mathbf{P}\left(S_{k}^{(a)}>0\right)-C T^{2} c_{n(a)} \sum_{k=T^{6} n(a)}^{\infty} \frac{1}{k c_{k}} \\
& \geqq \sum_{k=T^{6} n(a)}^{\infty} \frac{1}{k} \mathbf{P}\left(S_{k}^{(a)}>0\right)-C T^{2-6 / \alpha},
\end{aligned}
$$

where in the last step we used the fact that $\left\{c_{n}\right\}$ is regularly varying with index $1 / \alpha$. Using this bound, we make sure that (24) yields

$$
\lim _{T \rightarrow \infty} \sum_{k=T^{6} n(a)}^{\infty} \frac{1}{k} \mathbf{P}\left(S_{k}^{(a)}>0\right)=0 \quad \text { uniformly in } a>0
$$

which is equivalent to (4). Thus, the proof is finished.

Acknowledgments. This work was carried out during the visits of the first author to Technische Universität München and Ludwig-Maximilians-Universität München and the visit of the second author to EURANDOM. Both authors are grateful to the above-mentioned institutions for their hospitality. The authors are also thankful to Ron Doney for drawing their attention to paper [11] which helped in simplifying the proof of the lemma.

\section{REFERENCES}

[1] S. Asmussen, Applied Probability and Queues, Springer-Verlag, New York, 2003.

[2] A. A. Borovkov, Transient phenomena for random walks with nonidentically distributed jumps having infinite variances, Theory Probab. Appl., 50 (2006), pp. 199-213.

[3] O. J. Boxma And J. W. Cohen, Heavy-traffic analysis for the GI/G/1 queue with heavy-tailed distributions, Queueing Syst., 33 (1999), pp. 177-204.

[4] J. W. Cohen, Random walk with a heavy-tailed jump distribution, Queueing Syst., 40 (2002), pp. 35-73.

[5] H. Furrer, Risk Theory and Heavy-Tailed Lévy Processes, Ph.D. Thesis, ETH, Zurich, 1997.

[6] I. I. Gikhman And A. V. Skorokhod, Introduction to the Theory of Random Processes, W. B. Saunders Company, Philadelphia, 1969.

Copyright $@$ by SIAM. Unauthorized reproduction of this article is prohibited. 
[7] J. F. C. Kingman, The single server queue in heavy traffic, Proc. Cambridge Philos. Soc., 57 (1961), pp. 902-904.

[8] J. F. C. Kingman, On queues in heavy traffic, J. Roy. Statist. Soc. Ser. B, 24 (1962), pp. 383392.

[9] J. F. C. Kingman, The heavy traffic approximation in the theory of queues, in Proceedings of the Symposium on Congestion Theory, W. L. Smith and W. E. Wilkinson, eds., University of North Carolina Press, Chapel Hill, NC, 1965, pp. 137-159.

[10] Ju. V. Prokhorov, Transition phenomena in queueing processes. I, Litovsk. Mat. Sb., 3 (1963), pp. 199-205 (in Russian).

[11] W. E. PruitT, The growth of random walks and Lévy Processes, Ann. Probab., 9 (1981), pp. 948-956.

[12] S. Resnick And G. Samorodnitsky, A heavy traffic limit theorem for workload processes with heavy tailed service requirements, Management Sci., 46 (2000), pp. 1236-1248.

[13] A. I. Sakhanenko, On transient phenomena in random walks, Theory Probab. Appl., 49 (2005), pp. 354-367.

[14] K.-I. Sato, Lévy Processes and Infinitely Divisible Distributions, Cambridge University Press, Cambridge, UK, 2005.

[15] E. Seneta, Regularly Varying Functions, Lecture Notes in Math. 508, Springer, Berlin, 1976.

[16] F. Spitzer, Principles of Random Walk, Springer-Verlag, Berlin, 2001.

Copyright (c) by SIAM. Unauthorized reproduction of this article is prohibited. 